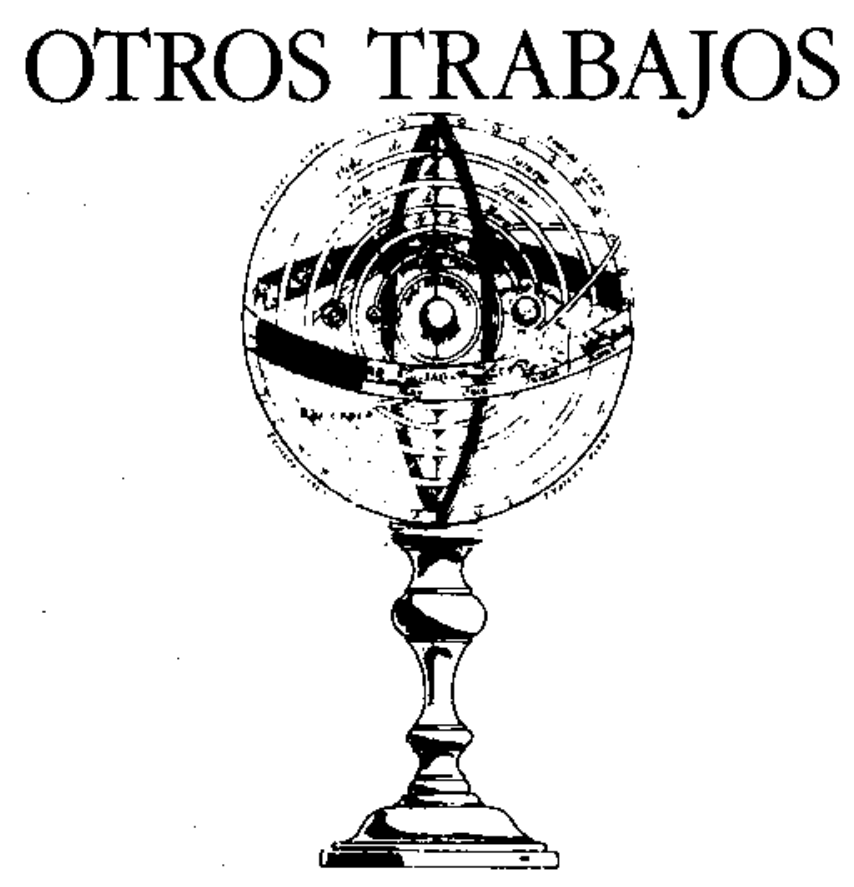

\title{
L'HOMME DU TROISIÈME MILLÉNAIRE FACE A L'INCERTITUDE." QUELLE FORMATION POUR AFFRONTER L'INCERTITUDE?
}

VIARD, J.

I. F. M. Université Joseph Fourier. B. P. 53 X. 38041 Grenoble Cedex

\section{SUMMARY}

In this paper we intend to establish the idea that a scientific formation can be an adequate background for future citizens to face the uncertainties of their own time. Howeves, this requires questioning the distorted view of science given by society and, more particularly, by education. This article analyzes the origin of the contradiction between scientists activity and its interpretation in society and education and presents some alternative proposals to overcome it.

1. L'EXPÉRIENCE SCIENTIFIQUE RÉFÉRENCE OBLIGEE DE TOUT PROJET DE FORMATION POUR LE TROISIEME MILLENAIRE

Si l'on veut bâtir une formation qui prépare à affronter I'incertitude, il est naturel de se référer à ceux qui, de part leur expérience, leur profession, leur vie quotidienne, sont précisément le plus confrontés à l'incertitude. 
Quels sont les aventuriers des temps modernes: ce ne sont ni les commerçants, ni les entrepreneurs, ni en premier lieu les techniciens mais les hommes de science. Le grand physicien des Etats-Unis mort récemment d'un cancer, Richard Feynman parle à propos de la science: "de la plus grande aventure que le genre humain ait jamais entreprise" (Feynman 1979).

Deux exemples pour illustrer cette affirmation:

- Le premier emprunté au passé: la naissance de la science moderne en Occident au 16ème siècle avec Galilée, Descartes.

Un historien des sciences résume cette époque ainsi: «De la physique du sens commun à celle du doute» (Rosmorduc 1985). Le doute devient en effet la règle de conduite, il remplace les certitudes et le consensus qui règnaient dans la pensée occidentale depuis près de deux millénaires. Cela se traduit par une formidable bataille idéologique.

- Plus proche de nous un exemple d'actualité: il a fallu quarante-six ans, depuis la première découverte du phénomène en 1911 jusqu'en 1957, pour bâtir une théorie pleinement satisfaisante de la supraconductivité. Trente ans plus tard, la découverte de nouveaux matériaux supraconducteurs à une température totalement inattendue impose de remettre en chantier une nouvelle théorie.

Ainsi on pourrait en conclure que pour développer l'intelligence définie communément comme «la faculté de s'adapter à des situations nouvelles", il suffit de $s$ 'inspirer de l'expérience et des méthodes de la science. Mais les choses ne sont pas aussi simples qu'il y paraît pour les raisons suivantes: l'image que l'on a du scientifique et du technicien dans la «société civile» n'est pas celle que l'on vient de donner. Ainsi, quand un scientifique exprime publiquement ses doutes sur l'étendue de son savoir ou la fjabilité de sa technique, on ne le croit pas. On estime qu'il ment ou qu'il est un mauvais scientifique ou technicien.

\section{Deux exemples:}

- Quand le professeur Luc Montagnier indique solennellement qu'au vu des connaissances actuelies il est peu probable que dans la décénie on fabrique un médicament efficace contre le Sida, il n'est pas cru et considéré comme un pessimiste.

-Quand, à plusieurs reprises, des ingénieurs de l'entreprise qui fabrique les propulseurs à poudre de la fuscé Challenger indiquent qu'il y a de gros problèmes avec les joints, que l'on court a la catastrophe en maintenant le vol prévu, on ne les écoute pas.

Autrement dit, on a l'idée magique d'une science surhumaine qui aurait sur-le-champ réponse à toutes les questions et d'une technique qui serait invulnérable. L'idée que la science soit une activité humaine comme une autre, c'est-à-dire difficile, faite de nombreux échecs mais capable d'efficacité dans la mesure même où ces échecs ont été acceptés, analysés et compris, l'idée que la technique est une entreprise fragile, et d'autant plus fragile que les risques encourus sont ignorés, cette idée est totalement rejetée.

Mais il y a plus grave, c'est l'image que la science donne d'elle-même dans l'enseignement:

Comme le fait remarquer un enseignant de mathématique de l'université Joseph Fourier, Marc Legrand (1989): si l'activité scientifique se caracterise par le doute, l'examen critique, la remise en cause des consensus par la confrontation des idées ou l'expérience, la complémentarité des points de vue, il en va tout autrement dans l'enseignement: «la situation d' enseignement (classique) est un oasis de certitude où le doute est bannin. Ici c'est le règne du définitif, de l'accord unanime sur la doctrine établie et parallèlement de la concurrence individuelle. On n'enseigne que les théories qui marchent et qui ne posent plus aucune question, celles dont la validité ne saurait faire le moindre doute. Il n'y a point de place pour des interprétations divergentes voire contradictoires, il faut aller droit au but en évitant les questions inutiles c'est-à-dire assimiler le maximum de connaissances et de connaissances sûres dans le minimum de temps. Ce n'est qu'au terme des études pour ceux qui auront eu la patience d'attendre et qui se seront illustrés dans l'exercice précédent que dans le meilleur des cas, les choses intéressantes commenceront avec l'accès à la recherche. Pour les autres, c'est I'exclusion.

Les élèves ou les étudiants ne s'y trompent pas, qui attachent peu d'importance aux démonstrations, à la validation des connaissances: à quoi bon démontrer des choses évidentes depuis des années et tenues pour vraies par ceux qui savent, qui ont l'expérience et les moyens techniques de la vérification. En définitive de l'école maternelle à I'université une affirmation est tenue pour vraie "parce que le mâtre ou le professeur l'a dite». C'est une phrase que l'on entend à l'universite. Le maître est celui qui dit le vrai et le faux.

On arrive ainsi àce paradoxe que c'est dans l'enseignement des sciences dites exactes, principales pourfenderesses de l'argument d'autorité, que cet argument s'impose avec le plus de force et que l'exercice de l'intelligence critique est le plus réduit. En 1983 à Grenoble un tiers des étudiants de la licence de physique, section recherche, ont demandé à leurs enseignants un enseignement qui fasse davantage appel à leur intelligence et ne se limite à un simple exercice de technique calculatoire. Cette requête, visiblement incomprise, est restée sans réponse.

\section{DEUX RAISONS PROFONDES DE LA SI- TUATION PRECECENTE}

Quelle est l'origine de cette contradiction entre l'activité de connaissance et sa traduction dans la société et l'enseignement de masse.

2. 1. L'une des raisons de la situation précédente a pour origine un choix idéologique 
A l'origine de cette situation il y a évidemment un choix idéologique et un choix de société résolumment élitiste qui n'a jamais été remis en cause depuis les débuts de la société industrielle. Taylorisme et élitisme sont des frères jumeaux. A l'affirmation «vous n'êtes pas là pour penser» du cadre de l'entreprise taylorienne répond le discours de l'enseignant élitiste: «yousn'êtes pas là pour comprendre mais pour apprendre."

En clair la situation actuelle dans I'enseignement qui fait des enseignés de simples consommateurs de connaissance et de savoir et les tient rigoureusement à l'écart de toute activitê de production et de création de ce savoir n'est pas le fait du hasard ou de l'ignorance mais d'un choix délibéré. Un des meilleurs promoteurs de l'élitisme au 19 ème siècle Emest Renan est parfaitement conscient des conditions indispensables d'un enseignement de qualité: Ia liberté de penser et la confrontation des idées, et il tient un discours très moderne avec lequel nous pourrions être en plein accord: "Ill n'y a pas de fort développement de la tête sans liberté» [...] "Ce qui importe à la jeunesse qui suit les cours de l'enseignement supérieur, c'est d'entendre des voix très diverses, $d^{\prime}$ assister au choc des opinions. Ce qu'on doit retirer de ces luttes', c'est moins un ensemble de doctrines fixes (il n'y en a guère dans les hautes régions de l'esprit humain) que l'exercice intellectuel, ... qui est le fruit de la discussion., (Renan 1982). Autrement dit ceux qui parlent aujourd'hui de "I'introduction du débat scientifique dans l'enseignement» (Brousseau 1981), (Legrand, Grenier, Richard 1984), n'ont tien inventé mais voilà, cet enseignement-là est réservé, pour Renan et beaucoup d'autres, à un petit nombre d'élus. Il y a un autre typed'enseignement. l'enseignement de masse dont la finalité est de "persuader' à I'homme du peuple d'être respectueux pour les grandes choses morales auquelles if coopère sans pouvoir toujours les comprendre".

Ce discours conserve aujourd'hui toute son actualité l'enseignement de masse touche aujourd'hui un public plus étendu et dure plus longtemps mais son esprit n'a pas changé comme en témoigne ces propos d'enseignants du secondaire ou de l'université entendus récemment: "On ne peut pas tout comprendre.» [...] «Si les étudiants cherchent à comprendre ils ne peuvent pas s'en sortir." "Pour apprendre tout ce qu'ils doivent savoir les étudiants doivent renoncer d comprendre ce qu' ils font.» Et enfin: "La compréhension est un fantasme d'intellectuel».

\section{2. La deuxième raison de cette situation est une raison de fond}

Penser est exaltant mais penser est difficile, pour tout le monde, pour l'enseigné comme pour l'enseignant. On connait la célébre phrase de Maxwell (1860): «Je connais la tendance de l'esprit humain d faire n' importe quoi plutôt que de penser.» Et Maxwell, dénonce déjà la tendance de ses collègues physiciens à «apprendre des formules» plutôt que de se livrer «au dur processus de la pensée».

Penser dérange et en particulier tout détenteur de pouvoir.
"Quelle chance pour ceux qui gouvernent que les hommes ne pensent pas !» (Adolphe Hitler).

Alors faut-il renoncer à enseigner, considérer que l'enseignant n'est efficace que pour ceux qui n'en ont pas besoin. Face à ce pessimisme nous avons recensé trois types d' expériences de formation qui donnent déjà des résultats encourageants.

\section{QUELQUES PROPOSITIONS ALTERNA- TIVES}

Un premier type d'expérience regroupe toutes celles qui visent à organiser la formation autour du développement de la personnalité de l'élève ou de l'étudiant.

Elle vise à accroître la prise de pouvoir de l'élève sur l'organisation de sa vie quotidienne, à accrôttre son initiative dans le déroulement de l'activité d'enseignement en lui permettant de prendre la parole autant qu'il le juge nécessaire, en développant au maximum ses capacités propres: sa curiosité, sa capacité à communiquer ses idées, ses sentiments dans le groupe auquel it appartient.

Ce type de pratique pédagogique développe très nettement à la fois le sentiment d'exister, la prise de responsabilité et l'aptitude à la socialisation, à la participation à un groupe. Le collège de la Vitle neuve de Grenoble en est une très bonne illustration. Interrogés sur le modèle d'entreprise dans laquelle ils souhaitent travailler plus tard les élèves de la Ville neuve ont indiqué qu'its souhaitent une entreprise dans laquelle la direction tienne le plus grand compte de l'avis de ses employés.

En conclusion remarquera que ce type de projet donne des résultats très intéressants quant au développement de la personnalité des élèves qui s'exprime très clairement dans la revendication précédente, toutefois ce projet ne remet nullement en question le rapport de l'élève au savoir. La connaissance transite toujours par le canal obligé du maître vers l'élève qui conserve dans ce domaine le pouvoir essenticl de dire le vrai et le faux. La condition principale de l'apprentissage reste «la relation confiante» entre le mấtre et l'élève.

Un autre type d'expérience regroupe toutes celles qui recherchent délibérément la rupture de la relation traditionnelle de dépendance enseigné-enseignant en sortant l'élève du contexte scolaire sans toutefois l'abandonner totalement à Iui-même ou en accompagnant I'élève đans son départ de l'institution lorsque cette sortie lui est imposée.

Le prototype de toutes ces expériences maintenant nombreuses est l'expérience «nouvelles qualifications» ou I'expérience Schwartz. Dans ce projet le formateur n'est plus l'initiateur obligé du processus d'apprentissage. L'origine du processus d'apprentissage réside dans la mise en présence de l'apprenant de problèmes existentiels et la mise en question qui en résultera de ses connaissances actuelles. L'insuffisance des réponses qu'il sera dans I'immédiat en mesure d'apporter suscite- 
ra le désir d'apprendre et donnera sens à une formation ultérieure ou immédiate. Ce processus est en même temps l'expérience d'une formidable autononie: celle de se poser à soi-même des questions que personne ne vous a suggérées et celle de rechercher ensuite par ses propres moyens la réponse à ces questions.

Le rôle du formateur s'il est plus discret n'en est pas moins indispensable. Le formateur est témoin du questionnement, il enregistre les interrogations, il guide le formé dans la formulation de ses questions puis dans la mise en place du type de réponse qu'il sera en mesure d'apporter. Enfin il peut le cas échéant répondre sur-lechamp à des demandes précises ou bâtir à partir des besoins recensés un projet de formation personnalisée. Ce type de démarches a permis à certains jeunes en situation d'échec scolaire total de retrouver le goût d'apprendre au point de reprendre avec succès un cycle complet d'étude et à beaucoup d'autres de reprendre confiance en eux-mêmes et en leurs capacités d'affronter positivement l'existence.

Un troisième type d'expérience vise à modifier radicalement le rapport de l'étudiant au savoir mais tout en se situant à l'intérieur du cadre scolaire ou universitaire traditionnel. Sans sortir de l'institution il s'agit de transformer les rapports sociaux existant au sein du groupe constitué par les étudiants et l'enseignant entre d'une part les étudiants entre eux, les étudiants et le maître et les rapports existant entre chacun des intervenants et le savoir Iui-même.

Examinons ces modifications: Premièrement le mâtre n'est plus le détenteur du savoir. (Nous reviendrons sur cette affirmation qui peut parâitre fantaisiste). S'il n'est pas le détenteur du savoir, le mâtre n'est plus le médiateur obligé de l'acquisition du savoir. La médiation de cette acquisition peut venir dans ce cas pour l'étudiant soit de ses camarades, soit de l'expérience soit occasionnellement de l'enseignant à titre d'interlocuteur ordinaire et non plus privilégié. S'il n'est plus le détenteur du savoir le maître est de ce fait privé de l'un de ses pouvoirs fondamentaux celui de dire le vrai et le faux. $\mathrm{Si}$ le maître ne detient plus le savoir et s'il n'exerce plus le pouvoir de dire le vrai et le faux alors les rapports de séduction ou ce qui revient au même de rejet, qui régissent très souvent les relations maître-elève $n^{\prime}$ ont plus de raisons $d^{\prime}$ être. L'importance des rapports affectifs dans l'apprentissage est considérablement diminuée.

Une question se pose aussitôt, comment cela est-il possible, ne s'agit-il pas d'une farce grossière? Le maître dispose bien de connaissances supérieures à l'élève sinon comment pourrait-il enseigner et par ailleurs comment peut-il laisser dire n'importe quoi dans sa classe, laisser énoncer sans réagir n'importe quelle contre vérité. La réponse réside évidemment dans la modification du sens donné au savoir à acquérir. La connaissance à acquérir n' est pas un objet que l'élève peut s'approprier mais il s'agit d'une connaissance en acte qui se traduit pratiquement par une modification des représentations, des conceptions et de la pratique de l'éleve ou de la classe avant l'enseignement et de ce savoir en acte, le maître ne peut être le détenteur. Cette modification en profondeur de l'ensemble du système de connaissance d'un élève ne peut être faite que par l'ćlève Iui-même et seulement s'il est convaincu que cette modification est pleinement justifiée, c'est-à-dire s'il a les moyens de juger par lui-même de la validité ou de la non validité de telle ou telle affirmation et dans ce cas l'affirmation intempestive d'un jugement extérieur d'autorité non seulement n'a plus de raison d'être mais va à l'encontre de l'objectif visé.

On commence à percevoir quel sera le rôle du maître: il lui revient de proposer au groupe d'étudiants des situations problèmes (Brousseau 1981) dans lesquels le groupe s'impliquera réellement. Confronté à ce problème l'étudiant formera des conjectures à partir de ses connaissances initiales en vue de la résolution du problème (Legrand, Grenier, Richard 1984). Il formulera ces conjectures pour les communiquer à la classe en justifiant son choix par une argumentation et le groupe réagira à cette conjecture en se reconnaissant en elle ou en proposant des alternatives. Le maître devra alors gérer ce débat en se contentant d'enregistrer les propositions des uns et des autres touten conservant une stricte neutralité (Brousseau 1981). Lorsque l'ensemble des avis auront été enregistrés au vu de tous viendra le moment de la mise à l'épreuve des conjectures ou des anticipations au moyen de la recherche de contre-exemples s'il s'agit d'énoncess généraux ou en mettant en place une expérience s'il s'agit d'une discipline expérimentale (Viard 1989). Ensuite, si la conjecture a été écartée et reconnue comme fausse il faudra poursuivre la recherche, dans le cas contraire si l'anticipation a été confirmée par l'expérience on recherchera une confirmation de ce résultat en le rattachant au reste du savoir admis, en mettant en place une démonstration. Si l'ensemble des connaissances dont disposent les élèves ne permettent pas de déboucher sur une solution le maître pourra être amené à introduire une notion nouvelle, mais même dans ce cas, qui se rapproche de la procédure habituelle, il y a une différence essentielle dans la mesure où une vraic question précède la réponse (Brousseau 1981).

On remarquera que cet enseignement utilise des outils identiques à ceux de l'enseignement traditionnel comme le problème, l'expérimentation, mais ces éléments ont changé de statut: le problème est le moyen utilisé habituellement après un enseignement pour vérifier l'efficacité de cet enseignement, ici le problème se situe au début du processus d'apprentissage et sa fonction est de susciter des questions et des actions en réponse à ces questions dans le but de construire un savoir nouveau. I' expérience est classiquement le moyen d'illustrer une théorie déclarée vraie par l'enseignant, ici l'expérience est le moyen de valider ou d'invalider les anticipations des étudiants (Viard 1991). Le statut du vrai et du faux cst également modifié comme celui de la réussite et de l'échec, l'un et l'autre perdent leur caractère de référence absolue: l'important n'est pas qu'une conjecture soit vraic ou fausse mais qu'elle existe et soit clairement formulee. Même fausse elle aura permis, une fois écartée, un progrès de la connaissance. De même l'échec d'unc tentative dans la résolution d'un problème est partie intégrante du processus de résolution puisqu'aucune solution n'est donnée à priori. 
Fn rósumé, en utilisant des ingrédients anciens: pro* blème, expérience, le processus qui vient d'être décrit, introduit dans la pratique des étudiants, pendant le cours, ce que Daniel Gil et J. Carascosa (1985) appellent un «changement méthodologique». Ils ont montré que ce changement méthodologique était une des conditions nécessaires du changement conceptuel, dans la mesure ò cette pratique du doute, de la contradiction, de l'interrogation, de la tentative, «implique, fondamentalement l'ébranlement de la confiance dans les "évidences du sens commun"». Un enseignement fait d'évidences fondées sur Ia seule autorité du professeur conforte, comme l'ont souligné I. A. Halloun et D. Hestenes(1985), la confiance dans les conceptions de sens commun. Le renoncement à l'autonomie intellectuelle, au jugement critique par soumission à l'autorité de l'institution, incarnée par le professeur, entraîne la même soumission à l'autorité non moins réelle du sens commun. Mais heureusement l'inverse est vrai également: le déve. loppement de l'autonomie intellectuelle dans un domaine permet ensuite de s'affranchir des autres dépen. dances.

Il y a évidemment un élément nouveau, c'est l'introduction đu débat d'idée argumenté qui constitue l'étappe centrale du processus, c'est ce débat qui permet de faire des connaissances initiales des étudiants les matériaux à partir desquels sera produite la nouvelle connaissance et c'est également ce débat qui permet de faire des étudiants les artisans de la construction de leur propre savoir.

Il apparaît très clairement que ce savoir acquis est un savoir commun au groupe, un savoir social non seulement parce que partagé entre tous mais aussi parce que tous ont pris part à sa production.

On constatera que l'autonomie n'est pas seulement l'objectif ultime du processus mais le moyen de sa réalisation, un tel processus d'apprentissage suppose l'autonomie, même

\section{NOTES}

(1) Ce texte a fait l'objet d'une intervention à un collougue dì prospective, organisé en Janvier 1989 par l'Alliance Universitaire de Grenoble, d'où le titre.

(2) CNAM: Conservatoire National des arts et Métiers. Organisme national français de formation pour adultes. si cette autonomie va en se renforçant. Comment fonctionne cette autonomic?

On remarquera que le moteur du processus d'apprentissage est la manifestation de l'écart entre la connaissance intiale du groupe et la connaissance nécessaire à la résolution du problème qui lui est soumis et la mesure de cet écart induira en retour une transformation de la connaissance initiale. Ce constat permet d'ćtablir un parallèle entre ce type d'enseignement et les nouvelles machines inspirécs du fonctionnement du cerveau humain que sont les réseaux de neurones formels. La propriété la plus intéressante des réscaux de neurones est leur capacité d'auto-apprentissage, la capacité d'acquérir par eux-mêmes de nouvelles connaissances qui les dis. tingue des ordinateurs les plus perfectionnés. Cot apprentissage se fait par la confrontation entre l'information produite par le réseau de neurones en sortie et l'information attenduc. De cette confrontation résulte une variable d'erteur qui induira une modification de l'architecture du réseau. De même dans un type d'enscignement fondé sur l'apprentissage autonome I'acquisition de nouvelles connaissances se traduira par une restructuration de l'ensemble des connaissances et non pas par la simple assimilation de nouveaux ćléments par simple écriture dans une case mémoire.

Ce type d'expérience est mis en œuvre actuellement à I'université Joseph Fourier, en premier cycle sous l'appelation de pratique du débat scienfique par Marc Legrand, en mathématique. EHe est pratiquée en mathématique également au CNAM $^{(2)}$ à Paris en formation continue, elle l'a ćté par l'auteur (de1986 à 1990) en formation continue également, au C. U. E. F. A. (3), mais en physique. Elle est pratiquée en physique également par des enseignants de I'enseignement secondaire dans le cadre de l'Institut de Formation des Maitres de Grenoble. Elle a également des équivalents aux États Unis à l'université sous l'appellation de processus dialectique d'enseignement (Halloun, Hestenes 1987).

(3) C. U. E. F. A. : Centre universitaire de formation pour adultes, situé à Grenoble

\section{REFERENCES BIBLIOGRAPHIQUES}

BROUSSEAU, G., 1981. Problèmes de didactique des décimaux. Recherches en didactique des mathématiques, Vol. II $n^{\circ} 1$, pp. 37-127.

FEYNMAN, R., 1979. Le cours de physique. InterEditions.

CARASCOSA, J., et GIL, D., I985. La «metodologia de lat superficialitat» de l'aprenentatge de les ciències, Enseñanza de las Ciencias, 3(2), pp. 113-120.

HALLOUN, I. A. et HiSTENES, D., 1985. Common sense 
concepts about motion, American Journal of Physics, $53(11)$, Nov. p. 1056

HALLOUN, I. A. et HESTENES, D., 1987. Modelling instruction in Mechanics, American Journal of Physics, 55(5), p. 455 May.

LAGRAND, M., 1989. La crise de l'enseignement: un problème de qualité. (Aleas Editeur: Lyon).

LEGRAND, M., GRENIER, D. et RICHARD, F., 1984. L'introduction du débat scientifique à l'intérieur du cours pour provoquer des processus de découvertes et de preuve. IIIème école d'été de Didactique des Mathématiques.

RENAN, E., 1982. La réforme intellectuelle et morale et autres écrits. (Albatros: Paris). Citć par M. J. Cuny et F. Petitdemange dans Le feu sous la cendre. Editions Cuny-Petitdemange.
RENAN, E., 1982. Questions contemporaines. Michel Lévy Frères éditeurs. (1870). Même source que précédemment.

ROSMORDUC, J., 1985. Une histoire de la physique et de la chimic. (Points Seuil: Paris).

MAXWELL, J. C., 1860. Conférence inaugurale au King's Collège de Londres. Cité par J. M. Lx́vy-Leblond et A. Butoli dans la Physique en questions. (Vuibert: Paris).

VIARD, J., 1989. Les aides à la didactique, outil d'introduction du débat scientifique en Physique. Actes des XIèmes Journécs Internationales sur I'éducation Scientifique. Chamonix.

VIARD, J., 1991. Trois tentatives pour enseigner la notion de force Newtonnienne à un groupe d'étudiants en formation continue, Petit $X, 25$, pp. 59-94.

\section{RESUMEN}

EI primer apartado de este trabajo lleva por título «La experiencia científica, referencia obligada de todo proyecto de formación para el tercer milenio», desarrollando la idea de que son los científicos quienes, por la naturaleza de su trabajo, mejor preparados están para enfrentarse a la incertidumbre, a la aventura que supone abordar lo desconocido. Sin embargo, la «sociedad civil» y, lo que es más grave, la propia enseñanza, proporcionan una visión absolutamente deformada de la ciencia: «si la actividad científica se caracteriza por la duđa, el examen crítico, la puesta en cuestión de los consensos mediante la confrontación de las ideas o la experiencia, la complementariedad de los puntos de vista (...) Ia situación de la enseñanza es un oasis de certidumbre en donde se ha desterrado la duda" (Marc Legrand 1989). FI segundo apartado analiza el origen de esta contradicción entre la actividad de los científicos y su traducción en la sociedad y en la enseñanza, avanzando razones de tipo sociológico: un elitismo que reduce a los estudiantes a simple consumidores de conocimientos y les mantiene rigurosamente al margen de toda actividad de producción y creación de esos conocimientos.

Por último, el tercer apartado recoge algunas propuestas alternativas, que se enmarcan claramente en una óptica constructivista, deteniéndose en particular en la necesaria transformación del papel del profesor y de las actividades habituales (problemas, prácticas de laboratorio...). Así, por citar un ejemplo, el papel de las prácticas no ha de ser el de ilustrar una teoría que el profesor introduce como verdadera, sino el de verificar o falsar las conjeturas de los estudiantes. 